\title{
5 Modelling the social and economic impact of an epidemic
}

\author{
Monika Bolińska, Pawet Dykas, \\ Tomasz Tokarski, and Rafat Wista
}

\subsection{Introduction}

The methods of analytical description of the spread of contagious diseases have been widely discussed in the scientific literature (see Murray, 2003; Ruan, 2007; Xiao, Ruan 2007; Fei-Ying, Wan-Tong \& Zhi-Cheng, 2015; Jardón-Kojakhmetov, Kuehn, Pugliese \& Sensi, 2021) that adopts the epidemiological model known as SIR (Susceptible-Infected/InfectiousRecovered/Removed), proposed by Kermack and McKendrick in 1927. The original SIR model ignores restrictions imposed on social and economic life to contain the spread of an epidemic and economic consequences of the epidemic and of those restrictions imposed to contain its spread. Bärwolff (2020) expanded the SIR model to include analyses of epidemic spread and subsidence. According to Bärwolff, the government introduces additional restrictions on socio-economic life when the number of people infected in the population exceeds the threshold set by the government. It also assumes that the introduction of stronger restrictions will slow the spread of the pandemic. However, he argues that a lockdown leads only to a displacement of the climax of the pandemic, but not really to an efficient flattening of the curve representing the number of infected people.

The effects of a rapid spread of a pandemic on economic growth were not analyzed in mainstream economic research in the past. The economic effects of HIV/AIDS in Asia (Bloom, Lyons, 1993) and in selected countries of Europe, Africa, North America and South America (Bloom, Mahal, 1995; Kambou, Devarajan, Over, 1992) were analyzed in the last two decades of the 20th century. For example, Bloom and Mahal, in their studies published in 1995 and 1997, argue that the HIV/AIDS epidemic had no material effect on the rate of growth of income per capita in 51 developed and industrialized countries of the world in the years 1980-1992. After two decades, Cuesta (2010) came to a similar conclusion about Honduras, the country most severely affected by the HIV/AIDS epidemic in South America.

The current scale and rate of spread of the COVID-19 pandemic, caused by a coronavirus, entails serious disturbances in social and economic life. The pandemic of 2020 represents the worst global health crisis since the 
Spanish flu that struck in 1918. In response to the chain of events observed, several measures are being presently considered. Alvarez, Argente and Lippi (2020) and Atkeson (2020) address the problem of optimization of the severity level of a lockdown. They use the SIR model under conditions of changing the economic activity of the population and enterprises. The importance of social distance is emphasized by Lik Ng (2020) who indicates adverse effects of a lockdown policy treated as the principal method preventing the spread of the pandemic. Research into trade-offs in public choices was also initiated in 2020. Aum, Lee and Shin (2020) analyze a trade-off between gross domestic product (GDP) and public health under pandemic conditions. They argue that a lockdown not only limits the spread of the pandemic but also mitigates the accumulated GDP loss in the long run. If no lockdown measures are taken during a pandemic, mass quarantining is necessary, leading to adverse economic effects. The self-employed who achieve relatively low income form the group exposed to the most severe consequences of a lockdown. Brock and Xepapadeas (2020) adopt an even wider perspective. They argue that continuous growth of consumption activities, capital accumulation and climate change could increase the exposure of society to the risk of infection. In their opinion, a policy preventing the spread of an epidemic should consist of two components. The first component includes short-term measures. The second component includes economic policies aimed at changing consumption patterns and addressing climate change.

Research projects described in the scientific literature also include studies into the effects of an epidemic on economic growth, employing neoclassical growth models. Cuddington (1993) used the Solow growth model (1956) to analyze the growth path of per-capita GDP in the context of the HIV/AIDS epidemic and its demographic consequences. The model used by him indicated a material risk of reduction in the GDP growth rate in Tanzania by the year 2010. Cuddington and Hancock (1994) adopted the same methodological approach to assess the effect of HIV/AIDS on the economy of Malawi. Delfino and Simmons (2005) identify significant empirical links between the health structure of the population and the productive system of an economy that is subject to infectious disease, in particular tuberculosis. Another neoclassical model of economic growth used in research into the effects of the spread of HIV on economic growth was proposed by Mankiw, Romer and Weil (1992). Lovasz and Schipp (2009) used that model to assess the effects of educational and health capital, and of the pace of epidemic spread on aggregate macroeconomic indicators. The effect of HIV is not the same in all countries, and even within individual countries. The economies characterized by developed healthcare infrastructures are capable of providing means that aim to prevent a rapid spread of an epidemic in its early phase. Additionally, Lovasz and Schipp, when analyzing the problem of accumulation of human capital under epidemic conditions, argue that a loss of human capital due to an epidemic does not always entail the same consequences. 
The education level and number of skilled workers and their outflow from manufacturing processes due to an epidemic affects the GDP growth rate to a varying extent. Similarly, the social capital stock is interrelated with economic growth under epidemic conditions.

The above outline of main topics of research into the impact of an epidemic on economic growth provides foundations to the epidemiologicaleconomic model proposed in this chapter. The proposed model incorporates restrictions imposed by the government on social and economic life in two alternative versions: in a gradual, continual manner as a function of the proportion of infected people in the population, and as a strict lockdown adopted abruptly by the government. The value of aggregate production is affected by the capital stocks, the rising percentage of infectious people that reduces investment and the rate of capital accumulation, and the scale of lockdown restrictions. The model proposed in this chapter is not strictly related or limited to the COVID-19 pandemic, as it is useful in analyzing the effects of any epidemic that leads to material social damage (a high percentage of infected and dead people, limited interpersonal contacts due to lockdown measures implemented) and economic losses (a drop in production caused by a collapse of aggregate demand and a reduction in the supply capacity of the economy, and consequently in the rate of capital accumulation).

\subsection{An epidemiological-economic model}

The epidemiological-economic model described below represents a compilation of the SIR (Susceptible-Infectious/Infected-Removed/Recovered) epidemiological model proposed by Kermack and McKendrick (1927) and the neoclassical model of economic growth proposed by Solow (1956).

The original SIR model does not include restrictions imposed on social and economic activity in response to the spread of an epidemic. For this reason, an analysis of the process of spread and subsidence of an epidemic was made using the SIR model as modified by Bärwolff (2020). Bärwolff assumes that governments impose restrictions on social and economic life when an epidemic begins to spread out of control (once the percentage of infected people exceeds a certain critical level defined in an arbitrary manner by the government). Bärwolff also assumes that the more restrictive the lockdown introduced, the slower the pace of epidemic spread.

Bärwolff's study is based on the assumption that the state introduces lockdown measures rapidly in an arbitrary manner (within a period or at certain time intervals). In our epidemiological-economic model, we assume that the level of lockdown severity is defined using a specific functional rule. Namely, we assume that the severity index of a lockdown is an analytical function of the percentage of the infected. If the percentage grows, the government does not use arbitrary criteria but follows the rule described by the function when imposing restrictions on social and economic life. 


\section{Monika Bolińska et al.}

\subsubsection{The epidemiological module}

We consider two scenarios when analyzing the spread and subsidence of an epidemic. Like in the original SIR model, we consider a scenario wherein the government has no access to a vaccine (preventing the disease spread) and a scenario wherein the government has a vaccine.

In the scenario with the government having no access to a vaccine, we assume that the spread of epidemic is described by the following differential equations:

$$
\left\{\begin{array}{l}
\Delta S_{t}=-\beta \kappa_{t} S_{t-1} I_{t-1} \\
\Delta I_{t}=\beta \kappa_{t} S_{t-1} I_{t-1}-\gamma I_{t-1} \\
\Delta H_{t}=\gamma b I_{t-1} \\
\Delta D_{t}=\gamma(1-b) I_{t-1}
\end{array}\right.
$$

where $S_{t} \in(0,1)$ represents the percentage of susceptible people on day $t$ (for $t=1,2, \ldots), I_{t} \in(0,1)$ represents the percentage of the infected, $H_{t} \in(0,1)$ represents the percentage of the recovered (the recovered are not eventually included in the group of the susceptible), and $D_{t} \in(0,1)$ represents the percentage of the dead. ${ }^{1}$ We also assume that $\beta, h \in(0,1), \gamma \in(0, \beta)$ and $\kappa_{t} \in[0,1]$ in consecutive days $t=1,2, \ldots$. The parameter $\beta$ in the system of equations (5.1) describes the pace of epidemic spread, $\gamma$ represents the percentage of infected people who either recover or die, and $h$ represents the mortality rate among the infected. The parameter $\kappa_{t}$ that can vary in its value in time (like in the original study of Bärwolff from 2020) represents an indicator of restrictions imposed on social and economic life on consecutive days of epidemic duration. If the parameter equals 1 , the government does not impose any restriction on social and economic life in response to the epidemic. If $\kappa_{t}=0$, a full lockdown is imposed. The lower the value of the $\kappa_{t}$ indicator, the stricter the lockdown imposed. Additionally, the lower the value of that indicator, the slower the spread of epidemic.

It follows from the first equation in the system (5.1) that a reduction in the percentage of the susceptible (that is $-\Delta S_{t}$ ) is directly proportional to the indicator of restrictions imposed on social and economic life $\left(\kappa_{t}\right)$, the percentage of the susceptible $\left(-\Delta S_{t-1}\right)$ and the percentage of the infected $\left(I_{t-1}\right)$. The second equation in the system (5.1) is interpreted so that an increase in the percentage of the infected (that is $\Delta I_{t}$ ) equals the difference between a reduction in the percentage of the susceptible (that is $-\Delta S_{t}$ ) and the percentage of the infected who recover or die $\left(\gamma I_{t-1}\right)$. Equations three and four in the system (5.1) imply that $h$ part of the infected recover and $1-b$ part of them die. Additionally, it follows from the second equation in the above system that the percentage of the infected $I_{t}$ rises as long as the

1 Certainly, on each day $t$ the equation is true: $S_{t}+I_{t}+H_{t}+D_{t}=1$. 
percentage of the susceptible $S_{t}$ is greater than the expression $\frac{\beta \kappa_{t}}{\gamma}$. Hence, restrictions imposed on social and economic life by the government (and described by a dropping value of the parameter $\kappa_{t}$ ) lead to a postponement of the initial day of a fall in the percentage of the infected. In the vaccination scenario, the SIR model is reduced to the following system of differential equations that represents an extension of the system of equations (5.1):

$$
\left\{\begin{array}{c}
\Delta S_{t}=\left\{\begin{array}{c}
-\beta \kappa_{t} S_{t-1} I_{t-1} \text { fort }<\tau+21 \\
-\beta \kappa_{t} S_{t-1} I_{t-1}-\varepsilon \rho \pi_{t-21} S_{t-21} \text { for } t \geq \tau+21
\end{array}\right. \\
\Delta I_{t}=\beta \kappa_{t} S_{t-1} I_{t-1}-\gamma I_{t-1} \\
\Delta H_{t}=\gamma h I_{t-1} \\
\Delta D_{t}=\gamma(1-b) I_{t-1} \\
\Delta P_{t}=\left\{\begin{array}{c}
0 \text { fort }<\tau+21 \\
\varepsilon \rho \pi_{t-21} S_{t-21} \text { fort } \geq \tau+21
\end{array}\right.
\end{array}\right.
$$

$P_{t} \in(0,1)$ in the system of equation (5.2) represents the percentage of effectively vaccinated people (that is people who are no longer susceptible to infection after their vaccination), $\tau$ represents the first day of vaccination, $\varepsilon \in(0,1)$ represents an indicator of vaccine effectiveness (that is the percentage of the vaccinated population that will not contract the disease), $\rho \in(0,1)$ represents the percentage of those who wish to receive the vaccine, and $\pi_{t} \in(0,1)$ (for consecutive days $t=\tau, \tau+1 \ldots$ ) represents the percentage of those who wish to receive the vaccine and are vaccinated until day $t$. We also assume that people effectively vaccinated develop immunity to the disease in 21 days after vaccination.

A modification in the system of differential equation (5.2) compared to the system of equation (5.1) can be reduced to the conclusion that beginning on day 21 after the first day of vaccination, the percentage of the susceptible is reduced by the percentage of effectively vaccinated people (that is by $\left.\varepsilon \rho \pi_{t-21} S_{t-21}\right)$.

When analyzing models without vaccination and with vaccination, we adopt two alternative scenarios of changes in the severity indicator of restrictions imposed on social and economic life $\kappa_{t}$. We assume that

$$
\kappa_{t}=1-I_{t-1}^{\sigma}
$$

or:

$$
\kappa_{t}= \begin{cases}1 & \text { for } \bar{I}_{G t}<\imath \\ \theta & \text { for } \bar{I}_{G t} \geq \imath\end{cases}
$$




\section{Monika Bolińska et al.}

where $\bar{I}_{G t}=\prod_{i=1}^{14} I_{t-i}$ represents a geometric moving average of the percentage of the infected in the most recent two weeks. Regarding the parameters $\theta, \sigma$ and $l$ in the equations (5.3) and (5.4), we assume that: $\theta, \imath \in(0,1)$, and $\sigma>0$.

We assume in equation (5.3) that if the percentage of the infected $I_{t}$ rises from 0 to 1 , the restriction severity indicator $\kappa_{t}$ drops from 1 to 0 , and if $\sigma \in(0,1)(\sigma>1)$, subsequent falls in the indicator $\kappa_{t}$, corresponding to identical rises in the percentage of the infected $I_{t}$, are increasingly bigger (smaller). ${ }^{2}$ Equation (5.4) implies that we consider a scenario wherein the government does not impose any restriction on social and economic life, if the geometric moving average of the percentage of the infected over the most recent two weeks does not exceed the percentage $t$. When that percentage is exceeded, the government imposes a lockdown and the indicator $\kappa_{t}$ drops abruptly from 1 to $\theta$.

The indicator of immunization coverage $\pi_{t}$ is described by the following equation:

$$
\pi_{t}=\frac{a t}{b+t}
$$

where $a, b>0$, and $t$ represents consecutive days of vaccination. That indicator of vaccination coverage (at $t$ increasing from 0 to $+\infty$ ) rises with a decreasing pace from 0 to $a .^{3}$

\subsubsection{The economic module}

We adopt the following assumptions about developments of basic macroeconomic variables in our economic module: ${ }^{4}$

1) The value of production on day $t$ (that is $Y_{t}$ ) is described by a modified Cobb-Douglas production function (1928) expressed by the formula:

$$
Y_{t}=\kappa_{t} K_{t}^{\alpha} L_{t}^{1-\alpha}
$$

2 This is because we obtain from a continuous function $f(x)=1-x^{\sigma}: f^{\prime}(x)=-\sigma x^{\sigma-1}$ and $f^{\prime \prime}(x)=(1-\sigma) \sigma x^{\sigma-2}$, and consequently for $\sigma>0: \forall x \in(0,1) f^{\prime}(x)<0, \sigma \in(0,1) \Rightarrow$ $f^{\prime \prime}(x)>0 \wedge \sigma>1 \Rightarrow f^{\prime \prime}(x)<0$.

3 This is because we obtain from a continuous function $f(x)=\frac{a t}{b+t}: f(0)=0, \lim _{t \rightarrow+\infty} f(t)=a$, $f^{\prime}(t)=\frac{a b}{(b+t)^{2}}>0 \wedge f^{\prime \prime}(t)=-\frac{2 a b}{(b+t)^{3}}(\forall t>-b)$.

4 Assumptions (1) and (2) refer directly to the model proposed by Solow in 1956, and assumptions 3 ) and 4) extend that model to include basic variables describing the functions of the labor market. 
where $\sigma \in(0,1)$ represents output elasticity $Y_{t}$ of capital input $K_{t}$. In function (5.5), we take into account both supply and demand factors affecting the value of production. The supply component (like in the original Cobb-Douglas production function) is described by the expression $K_{t}^{\alpha} L_{t}^{1-\alpha}$, hence if the epidemic did not strike, the value of production (like in the Solow model) would amount to ${ }^{5} K_{t}^{\alpha} L_{t}^{1-\alpha}$. We also assume that if the government imposes a lockdown and reduces the indicator of social and economic activity from 1 to $\kappa_{t} \in(0,1)$, the value of aggregate demand falls and (due to Keynesian multiplier effects) the volume of production also falls from a level of $K_{t}^{\alpha} L_{t}^{1-\alpha}$ to $\kappa_{t} K_{t}^{\alpha} L_{t}^{1-\alpha}$. Hence, a relative reduction in the volume of production caused by a fall in $\delta$. Like in the original model proposed by Solow, capital accumulation (daily, in a discrete time) is described by a differential equation in the following form:

$\Delta K_{t}=s \frac{Y_{t-1}}{365}-\delta \frac{K_{t-1}}{365}$

where $s \in(0,1)$ represents the savings-investment rate, and $\delta \in(0,1)$ represents the capital depreciation rate.

2) The value of demand for labor (and the number of currently employed people) is described by:

$L_{t}=\omega\left(1-I_{t}-D_{t}\right)\left(\frac{Y_{t}}{Y^{*}}\right)^{\phi}$

where $\omega, \phi \in(0,1)$, and $\mathrm{Y}^{*}>0$ represents the value of production in the Solow long-run steady state (that is at $\Delta K_{t}=0$ ). The parameter $\phi$ represents elasticity of demand for labor relative to the volume of production. $I_{t}$ and $D_{t}$ in equation (5.7) represent (like in the epidemiological module of the proposed model) percentages of the infected and those who died of the epidemic.

It follows from equation (5.7) that in our model, if the epidemic did not strike, at production rising from $Y_{t}<Y^{\prime \prime}$ to $Y^{\prime \prime}$, the percentage of the employed would rise from a level of $\omega\left(\frac{Y_{t}}{Y^{*}}\right)^{\phi}$ to $\omega$. In the time of epidemic, the percentage of the employed represents $\left(1-I_{t}-D_{t}\right)$ part of the demand for labor, because the infected and dead (certainly) do not work.

3) The unemployment rate $u_{t}$ is (by definition) described by the formula:

$u_{t}=1-\frac{L_{t}}{w}$

5 To simplify notation, we assume that the total factor productivity on each day $t$, described by the formula $\frac{Y_{t}}{K_{t}^{\alpha} L_{t}^{1-\alpha}}$, equals 1 . This has no effect on the scope of applicability of the below discussion. 
where $w \in(0,1)$ represents the percentage of the professionally active. We assume implicitly that on day $t=1$ the population amounted to 1 , and on consecutive days equalled $1-D_{t}$ while the number of professionally active people amounted to $w\left(1-D_{t}\right)$.

It follows from equations $(5.5-5.8)$ that in the Solow long-run steady state (i.e. at $\left.\Delta K_{t=0}\right): L^{*}=\omega, K^{*}=\omega\left(\frac{s}{\delta}\right)^{\frac{1}{1-\alpha}}, Y^{*}=\omega\left(\frac{s}{\delta}\right)^{\frac{\alpha}{1-\alpha}}$ and $u^{*}=\frac{w-\omega}{w}$, where asterisks next to consecutive variables indicate their values in the steady state of the economic growth model analyzed here.

The following system of differential equations is obtained from equation (5.5)-(5.8):

$$
\left\{\begin{array}{l}
Y_{t}=\kappa_{t} K_{t}^{\alpha} L_{t}^{1-\alpha} \\
\Delta K_{t}=s \frac{Y_{t-1}}{365}-\delta \frac{K_{t-1}}{365} \\
L_{t}=\omega\left(1-I_{t}-D_{t}\right)\left(\frac{Y_{t}}{Y^{*}}\right)^{\phi} \\
u_{t}=1-\frac{L_{t}}{w} \\
U_{t}=\sqrt[4]{\left(1-I_{t}\right) \kappa_{t} \frac{u_{N t}}{u_{t}} \frac{Y_{t}}{Y_{N t}}}
\end{array}\right.
$$

where $u_{N}$ and $Y_{N}$ represent (respectively) an unemployment rate and a production value that would be recorded if the epidemic did not strike (that is in a scenario wherein on each day $t=1,2, \ldots$ the percentage of the susceptible $S_{t}$ would equal 1 ).

The last equation in system (5.9) describes the social utility function $U$. The function represents a geometric average of the indicator of social and economic activity $\kappa$, the percentage of the susceptible $1-I$, the ratio of the unemployment rate under non-epidemic to that rate under epidemic conditions $\left(u_{N} / u\right)$ and the ratio of production under non-epidemic conditions to production under epidemic conditions $\left(Y / Y_{N}\right)$. The function of social utility $U_{t}=\sqrt[4]{\left(1-I_{t}\right) \kappa_{t} \frac{u_{N t}}{u_{t}} \frac{Y_{t}}{Y_{N t}}}$ takes into account both social (described by the indicator $\left.\kappa_{t}\right)$ and health $\left(1-I_{t t}\right)$, and economic $\left(u_{N t} / u_{t}\right.$ and $\left.Y_{t} / Y_{N t}\right)$ consequences of the epidemic.

Additionally, the social utility function $U_{t}$ assumes values from the interval $[0 ; 1]$. If the epidemic did not strike, $\kappa_{t}=1-I_{t}=\frac{u_{N t}}{u_{t}}=\frac{Y_{t}}{Y_{N t}}=1$, and hence $U_{t}=1$. The lower values are assumed by function $U$, the higher 
are aggregate social, health and economic costs of the epidemic. During a full lockdown (that is at $\kappa=0$ ), the value of social utility function falls to 0 .

\subsection{Calibrated model parameters}

\subsubsection{Parameters of the epidemiological module}

We assume that the infection lasts for 14 days on average. Hence, the parameter $\gamma$ in the epidemiological module is selected at the level of $\gamma=1 / 14 \approx 0.071429$.

The parameter $\beta$ is calibrated so that peak incidence, if the government does not impose any lockdown, falls on day 365 of the epidemic. Hence the parameter equals 0.1066 in consecutive versions of numerical simulations.

We also assume that the mortality rate among the infected amounts to $2 \%$, hence $h=0.98$. We assume that one person per million was infected on day one of the epidemic, that is $I_{1}=10^{-6}$.

When analyzing the equation of social and economic activity indicator $\kappa_{t}=1-I_{t}^{\sigma}$, we assume $\sigma$ equals 0.5 (if the government imposes severe restrictions to contain the epidemic) or 1 (if a liberal approach is adopted). When we use the function $\kappa_{t}=\left\{\begin{array}{ll}1 & \text { for } \bar{I}_{G t}<\imath \\ \theta & \text { for } \bar{I}_{G t} \geq \imath\end{array}\right.$ to describe restrictions imposed by the government to contain the epidemic, we assume that the government adopts a lockdown when the geometric moving average of the percentage of the infected $\bar{I}_{G t}$ exceeds $l=0.5 \%$ and then social and economic activity will be reduced by $15 \%$ (that is $\theta=0.85$ ). If the government adopts a liberal approach to the epidemic, we assume $l=1 \%$ and $\theta=0.95$.

When analyzing the models with vaccination, we assume that vaccines are administered as of day 300 of the pandemic. We also assume that a percentage $\rho=48 \%$ of the population wish to receive the vaccine and the effectiveness of vaccination $\varepsilon$ equals $95 \%$.

We make two alternative assumptions about the dynamics of daily immunization coverage in the population $\pi_{t}$ :

- first, we assume that the parameters $a$ and $b$ in the indicator of immunization coverage are such that the indicator equals $1 \%$ on day 7 of vaccination and $2 \%$ on day 100 . Hence, we obtain: $\frac{7 a}{b+7}=0.001$ and $\frac{100 a}{b+100}=0.002$ which gives (in line with the Cramer's rule): $a=\frac{93 \cdot 0.001 \cdot 0.002}{100 \cdot 0.001-7 \cdot 0.002} \approx 0.00216$ and $b=\frac{700 \cdot(0.002-0.001)}{100 \cdot 0.001-7 \cdot 0.002} \approx 8.140$. The scenarios are referred to below as scenarios with slow progress in immunization coverage of the population; 
- second, we assume that $\pi_{7}=0.001$ and $\pi_{100}=0.006$. In this case, the Cramer's formula produces: $\mathrm{a} \approx 0.00962$ and $\mathrm{b} \approx 60.345$. The scenarios are referred to below as scenarios with rapid progress in immunization coverage.

\subsubsection{Parameters of the economic module}

The elasticity $\alpha$ of Cobb-Douglas production function (5.5) is calibrated at the level of 0.5 . We also assume a $20 \%$ savings-investment rate $s$ and a $5 \%$ capital depreciation rate $\delta$. The long-run capital output ratio $K^{\prime \prime} / Y^{\prime \prime}$ at the values of those parameters set as above equals 4 .

We assume the indicator of economic activity of the population $w=$ $46 \%$, that is similar to the value recorded in the EU states.

The parameter $\omega$ in the function of demand for labor (5.7) is calibrated at the level of 0.44 , and consequently the long-run unemployment rate equals about $4.35 \%$ at $w=0.46$. The parameter $\phi$ is selected so that under nonepidemic conditions, in an economy with an initial capital input $K_{1}$ representing $40 \%$ of capital in the Solow long-run steady state (that is $K^{*}$ ), the unemployment rate equals $10 \%$. Then, the elasticity of demand for labor $L_{t}$ relative to production $Y_{t}$ equals about 0.106 .

\subsection{Scenarios and numerical simulation results}

The numerical simulations discussed below include 12 scenarios of epidemic development. The first four of those scenarios give the government no access to a vaccine, and a vaccine is available in the remaining eight scenarios (see the statement in Table 5.1).

In the scenarios wherein the government has no access to a vaccine (scenarios I-IV), we assume that the government reduces the intensity of social and economic activity gradually, in line with a functional formula (5.3) (scenarios I and II) or that activity is restricted abruptly (scenarios III and IV). In scenarios I and III, the government imposes severe restrictions to contain the spread of the epidemic; in scenarios II and IV, the government adopts a liberal approach.

The scenarios with vaccination (V-XII) can be divided into those with slow progress (scenarios V-VIII) and those with rapid progress (IX-XII) in immunization coverage of the population. Scenarios V and IX assume that the government adopts a lockdown like in scenario I; scenarios VI and X assume a lockdown as in scenario II, etc.

The results of numerical simulations of epidemiological indicators in the extended SIR model (systems of equations (5.1-5.2)) in consecutive scenarios are contained in Table 5.2. Figures 5.1-5.4 represent curves of analyzed epidemiological variables. ${ }^{6}$

6 All epidemiological simulations are carried out for a five-year period while macroeconomic simulations for a three-year period. This is because curves of macroeconomic variables stabilize after three years. 


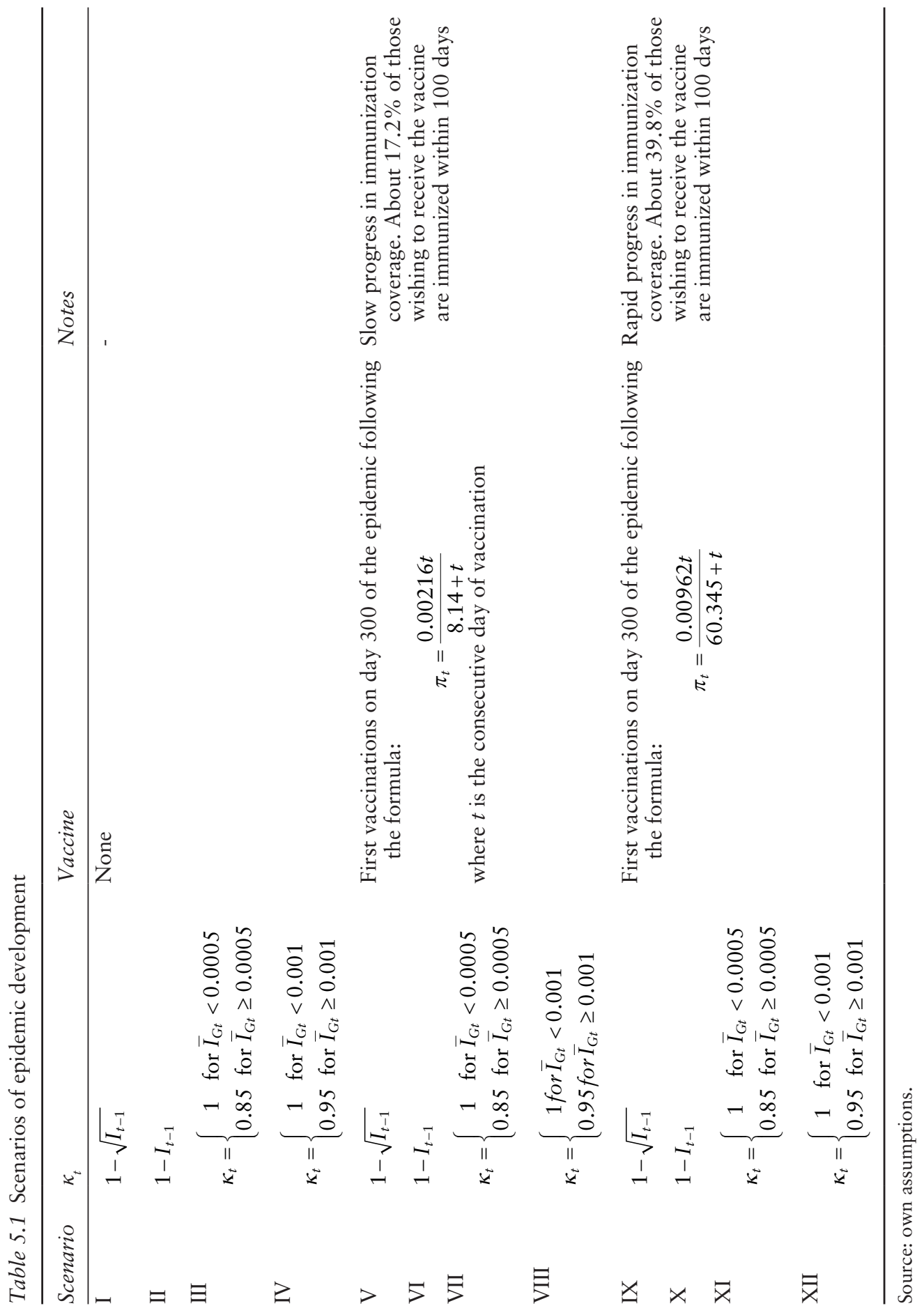


Table 5.2 Epidemiological indicators in consecutive scenarios

\begin{tabular}{llllllll}
\hline Scenario & \multicolumn{7}{l}{ Variable } \\
\cline { 2 - 7 } & $\kappa_{m}$ & $S_{m}$ & $\boldsymbol{I}_{M}$ & $\boldsymbol{H}_{M}$ & $\boldsymbol{D}_{M}$ & $\boldsymbol{P}_{M}$ & $\boldsymbol{T}$ \\
\hline I & 0.8347 & 0.5762 & 0.0273 & 0.4153 & 0.0085 & - & 391 \\
II & 0.9475 & 0.4566 & 0.0525 & 0.5326 & 0.0109 & - & 365 \\
III & 0.85 & 0.6043 & 0.0246 & 0.3878 & 0.0079 & - & 456 \\
IV & 0.95 & 0.4728 & 0.0490 & 0.5167 & 0.0105 & - & 383 \\
V & 0.8380 & 0.1485 & 0.0263 & 0.3374 & 0.0069 & 0.5072 & 381 \\
VI & 0.9480 & 0.1122 & 0.0520 & 0.4927 & 0.0101 & 0.3850 & 363 \\
VII & 0.85 & 0.1650 & 0.0189 & 0.2650 & 0.0054 & 0.5646 & 428 \\
VIII & 0.95 & 0.1181 & 0.0473 & 0.4649 & 0.0095 & 0.4075 & 378 \\
IX & 0.8389 & 0.0014 & 0.0260 & 0.2885 & 0.0059 & 0.7042 & 377 \\
X & 0.9481 & 0.0010 & 0.0519 & 0.4632 & 0.0095 & 0.5264 & 363 \\
XI & 0.85 & 0.0016 & 0.0167 & 0.1939 & 0.0040 & 0.8006 & 408 \\
XII & 0.95 & 0.0011 & 0.0468 & 0.4259 & 0.0087 & 0.5643 & 376 \\
\hline
\end{tabular}

The subscript $m$ indicates the minimum value of a variable, $M$ - its maximum value. $T$ - day of the greatest percentage of the infected.

Source: own calculations.

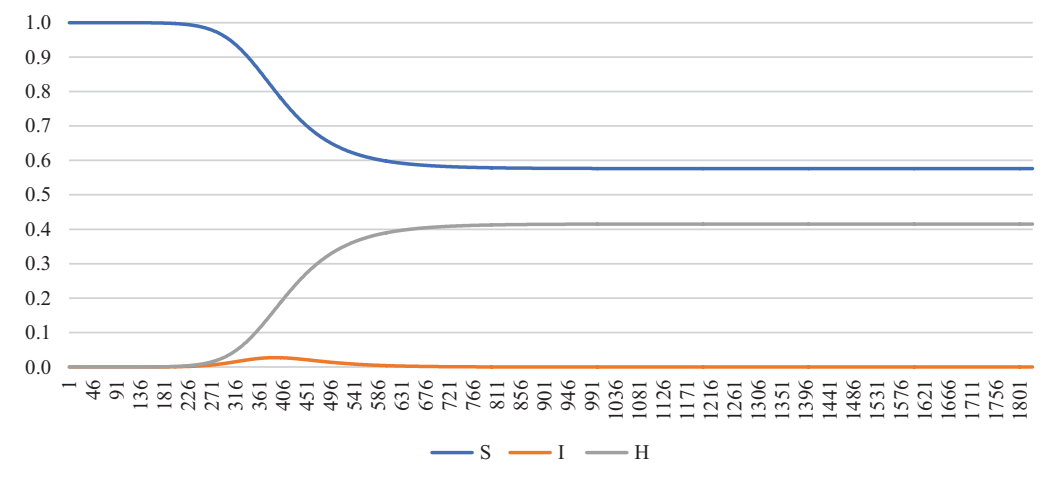

Figure 5.1a Curves of $S, I, H$ and $P$ in scenarios I, V and IX (at $\kappa_{t}=1-\sqrt{I_{t-1}}$ ) Scenario I. Source: own calculations.

The simulation results contained in Table 5.2 and Figures 5.1-5.4 lead to the following conclusions:

- if the government did not adopt any lockdown measures and had no access to a vaccine, the greatest percentage of the infected would be recorded (as already indicated) on day 365 of the epidemic. If the government has no access to a vaccine and imposes a severe lockdown, the peak will be postponed to day 391 (scenario I) or 456 (scenario II) of the epidemic. If a mild lockdown is imposed, the greatest number of the infected will be recorded on day 365 (scenario II) or 383 (scenario IV); 


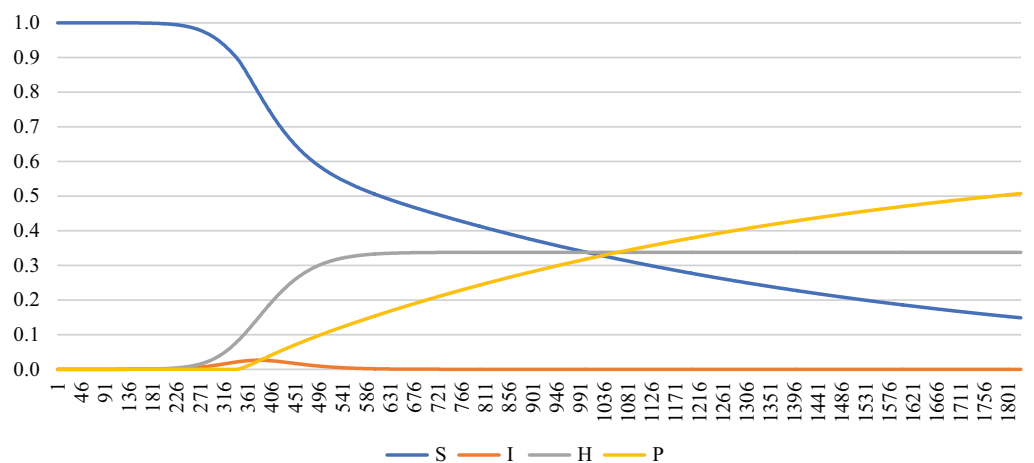

Figure 5.1b Curves of $S, I, H$ and $P$ in scenarios I, V and IX (at $\kappa_{t}=1-\sqrt{I_{t-1}}$ ) Scenario V. Source: own calculations.

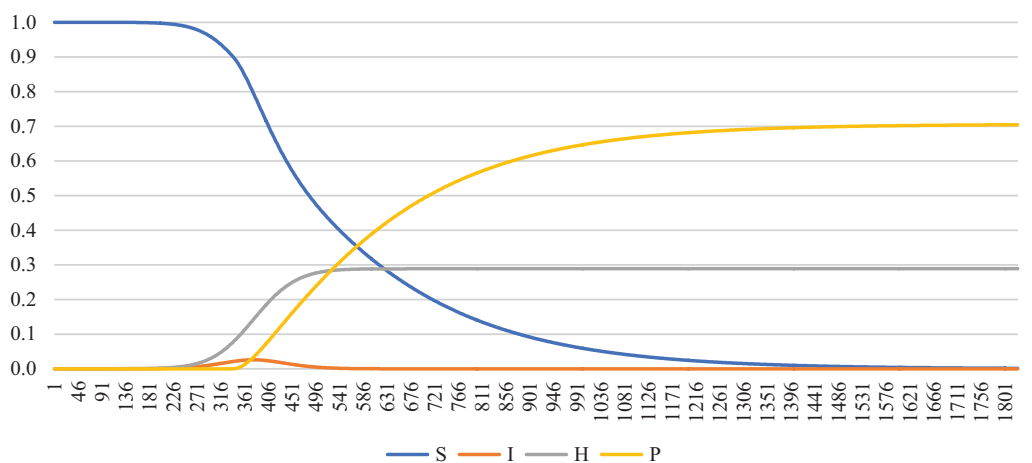

Figure 5.1c Curves of $S, I, H$ and $P$ in scenarios I, V and IX (at $\kappa_{t}=1-\sqrt{I_{t-1}}$ ) Scenario IX. Source: own calculations.

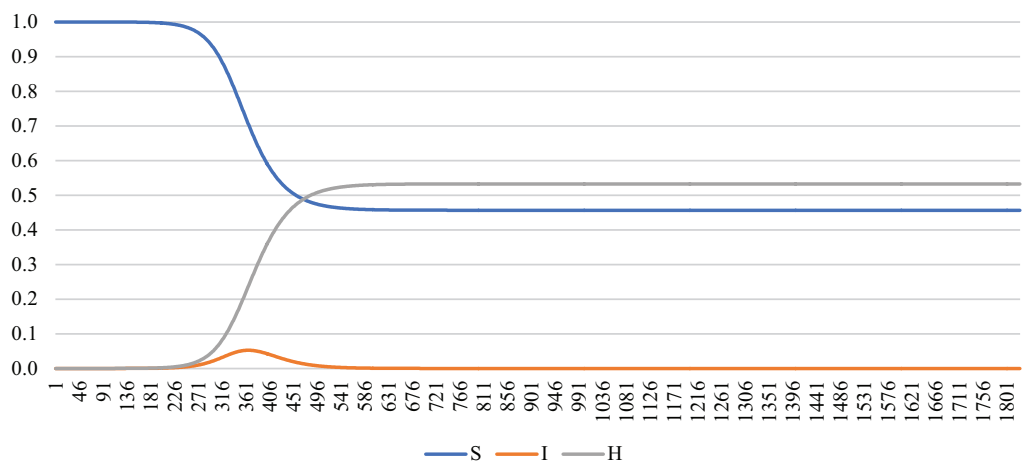

Figure 5.2a Curves of $S, I, H$ and $P$ in scenarios II, VI and X (at $\left.\kappa_{t}=1-I_{t-1}\right)$ Scenario II. Source: own calculations. 


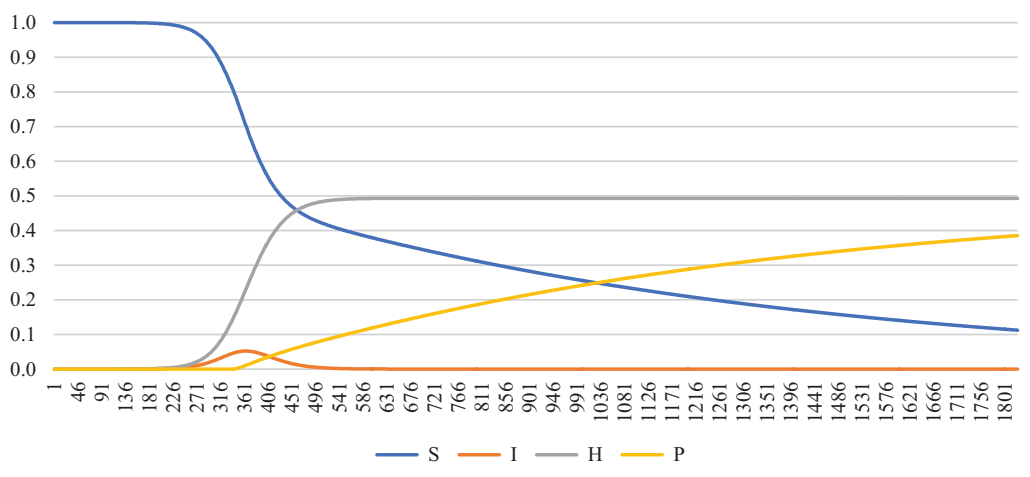

Figure 5.2b Curves of $S, I, H$ and $P$ in scenarios II, VI and X (at $\left.\kappa_{t}=1-I_{t-1}\right)$ Scenario VI. Source: own calculations.



Figure 5.2c Curves of $S, I, H$ and $P$ in scenarios II, VI and X (at $\left.\kappa_{t}=1-I_{t-1}\right)$ Scenario X. Source: own calculations.

- at slow progress in immunization coverage of the population (scenarios V-VIII), the greatest number of the infected is recorded between days 381 and 428 of the epidemic (if severe restrictions are imposed in response to the epidemic) or between days 363 and 378 (if a liberal approach to the epidemic is adopted). On the other hand, rapid progress in immunization coverage results in a postponement of epidemic peak to a date between days 377 and 408 of the epidemic (if severe restrictions are imposed in response to the epidemic), or between days 363 and 376 (if a liberal approach to the epidemic is adopted);

- in the scenarios wherein the government has no access to a vaccine, a maximum limitation of social and economic activity (at the peak of the epidemic) can reach $15-16.5 \%$ under conditions of a severe lockdown or $5-6.5 \%$ under conditions of a mild lockdown. The scenarios wherein 


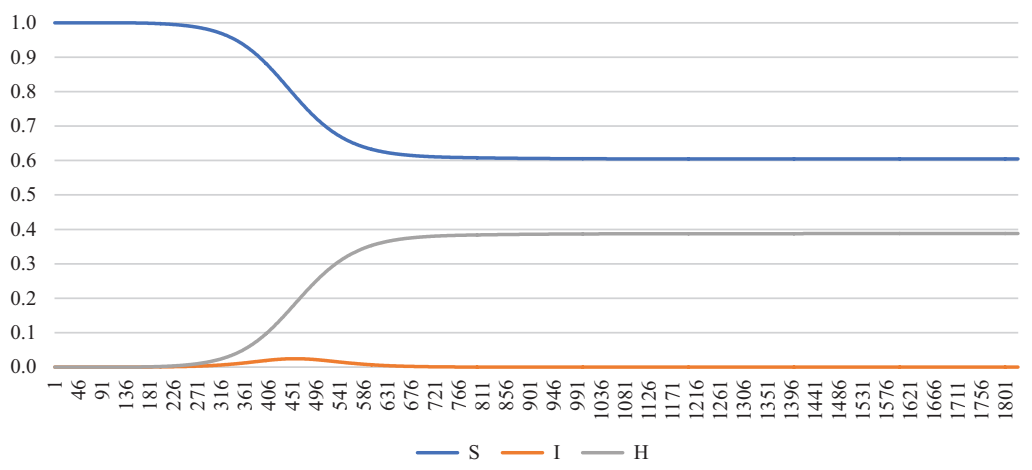

Figure $5.3 a$ Curves of $S, I, H$ and $P$ in scenarios III, VII and XI, at $\kappa_{t}=\left\{\begin{array}{c}1 \mathrm{dla} \bar{I}_{G t}<0.0005 \\ 0.85 \mathrm{dla} \bar{I}_{G t} \geq 0.0005\end{array}\right.$ Scenario III. Source: own calculations.

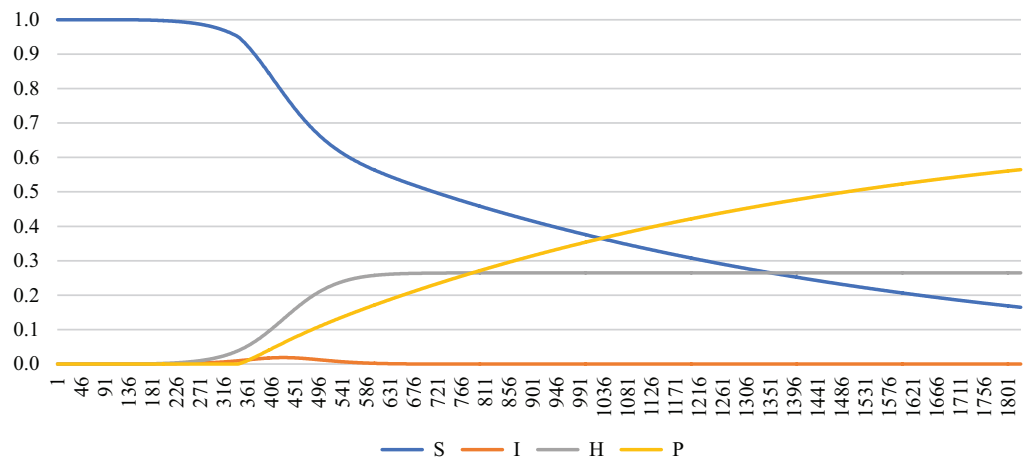

Figure $5.3 b$ Curves of $S, I, H$ and $P$ in scenarios III, VII and XI, at $\kappa_{t}=\left\{\begin{array}{c}1 \mathrm{dla} \bar{I}_{G t}<0.0005 \\ 0.85 \mathrm{dla} \bar{I}_{G t} \geq 0.0005\end{array}\right.$ Scenario VII. Source: own calculations.

the government uses vaccination (i.e. scenarios V-XII) have no significant effect on that parameter;

- if no vaccine is administered, the maximum percentage of infected people will reach $2.5-2.7 \%$ (severe restrictions in scenarios I and III) or $4.9-5.3 \%$ (liberal scenarios II and IV). In the case of slow progress in immunization coverage, that percentage will drop to about $1.9-2.6 \%$ under conditions of a severe lockdown or to $4.7-5.2 \%$ if a liberal approach is adopted. In the case of rapid progress in immunization coverage, that percentage will slightly fall; 


\section{Monika Bolińska et al.}

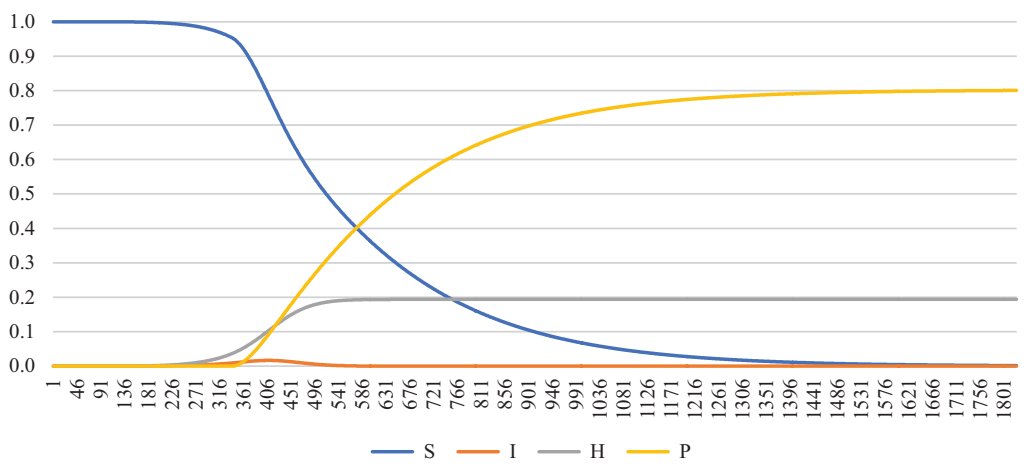

Figure $5.3 c$ Curves of $S, I, H$ and $P$ in scenarios III, VII and XI, at $\kappa_{t}=\left\{\begin{array}{c}1 \mathrm{dla} \bar{I}_{G t}<0.0005 \\ 0.85 \mathrm{dla} \bar{I}_{G t} \geq 0.0005\end{array}\right.$ Scenario XI. Source: own calculations.

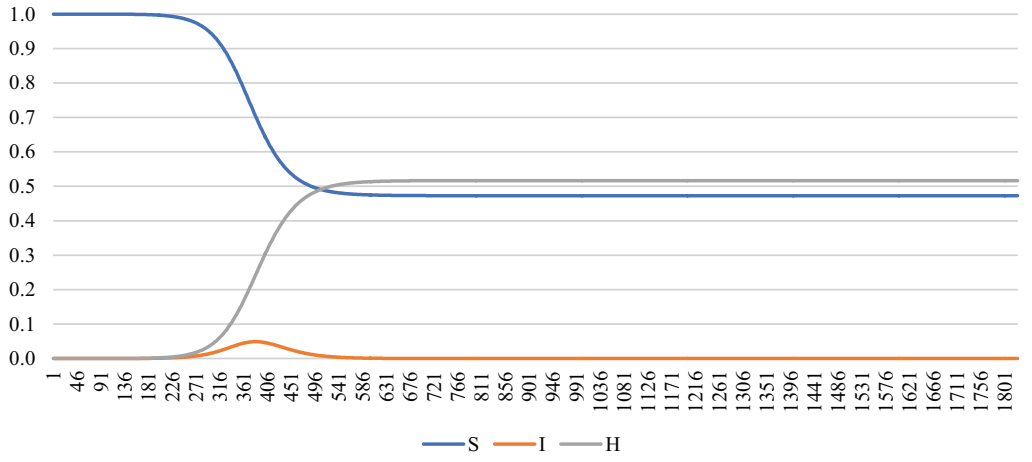

Figure 5.4a Curves of $S, I, H$ and $P$ in scenarios IV, VIII and XII, at $\kappa_{t}=\left\{\begin{array}{c}1 \mathrm{dla} \bar{I}_{G t}<0.001 \\ 0.95 \mathrm{dla} \bar{I}_{G t} \geq 0.001\end{array}\right.$ Scenario IV. Source: own calculations.

- in the scenarios without vaccination, the percentage of the susceptible (uninfected) will reach after the epidemic about 57.6-60.4\% under conditions of a severe lockdown or $45.7-47.3 \%$ under conditions of a mild lockdown;

- if the government has access to a vaccine but progress in immunization coverage is slow, the percentage of uninfected population (understood then as $S_{m}+P_{M}$ ) will reach $65.6-73.0 \%$ under conditions of a severe lockdown or $49.7-52.6 \%$ if a liberal approach is adopted;

- rapid progress in immunization coverage leads to an increase in those indicators to $70.6-80.2 \%$ (a severe lockdown) or $52.7-56.5 \%$ (a mild lockdown); 


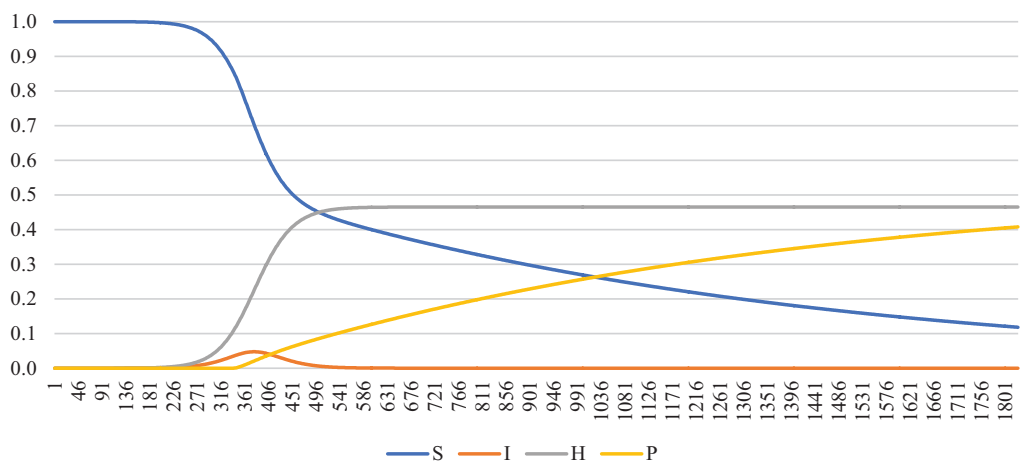

Figure 5.4b Curves of $S, I, H$ and $P$ in scenarios IV, VIII and XII, at $\kappa_{t}=\left\{\begin{array}{c}1 \mathrm{dla} \bar{I}_{G t}<0.001 \\ 0.95 \mathrm{dla} \bar{I}_{G t} \geq 0.001\end{array}\right.$ Scenario VIII. Source: own calculations.

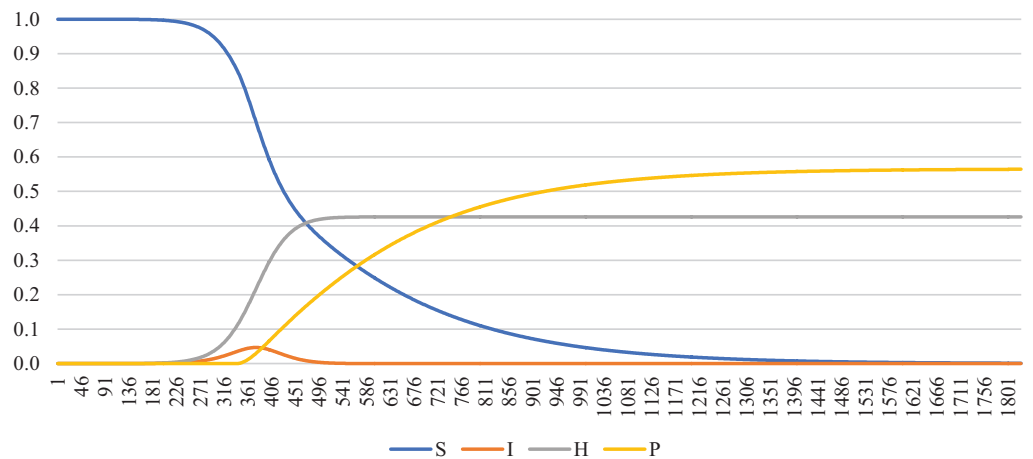

Figure $5.4 c$ Curves of $S, I, H$ and $P$ in scenarios IV, VIII and XII, at $\kappa_{t}=\left\{\begin{array}{c}1 \mathrm{dla} \bar{I}_{G t}<0.001 \\ 0.95 \mathrm{dla} \bar{I}_{G t} \geq 0.001\end{array}\right.$ Scenario XII. Source: own calculations.

- if no vaccine is administered, $7.9-8.5 \%$ of the population will die of the epidemic under conditions of a severe lockdown imposed by the government or $10.5-10.9 \%$ under conditions of a mild lockdown. Slow progress in immunization coverage will reduce those indicators to $5.4-6.9 \%$ (a severe lockdown) or $9.5-10.1 \%$ (a liberal approach). Rapid progress in immunization coverage will reduce the rate of mortality caused by the epidemic to $4.0-5.9 \%$ of the population (a severe lockdown) or $8.7-9.5 \%$ (a mild lockdown).

An analysis of the epidemic effect on the values of principal macroeconomic indicators (in the real economy sector) includes the scenarios described 
above in two versions. We consider values of those indicators in an economy conventionally termed "poorly developed" (with capital input $K_{1}$ representing $40 \%$ of the value of that variable in the Solow long-run steady state) and in a strongly developed economy (with $\left.K_{1}=0.9 K^{*}\right) 7$.

Selected results of numerical simulations are contained in Tables 5.3 (a poorly developed economy) and 5.4 (a strongly developed economy). Figures 5.5-5.7 depict curves of the social utility function $U_{t}$ in consecutive scenarios both in a poorly developed and in a strongly developed economy.

The simulation results contained in Tables 5.3-5.4 lead to the following conclusions:

- in a poorly developed economy that has no access to a vaccine, falls in production at peak incidence (measured by the indicator $\min _{t}\left\{\frac{Y_{t}}{Y_{N t}}\right\}$, where $Y_{N t}$ represents the value of production that could be achieved if the epidemic did not strike) will reach 18.6-19.9\% under conditions of a severe lockdown or $10.7-10.9 \%$ if a liberal approach is adopted. In a strongly developed economy, the falls are slightly smaller and reach (respectively) $18.3-19.7 \%$ or $10.4-10.6 \%$;

- slow progress in immunization coverage of the population combined with severe restrictions imposed in response to the epidemic will reduce falls in production to $17.9-19.5 \%$ in a poor economy or $17.7-19.3 \%$ in a wealthy economy. If a liberal approach to the epidemic is adopted, falls in production will reach (respectively) $10.5-10.6 \%$ or $10.2-10.3 \%$;

- rapid progress in immunization coverage has no material effect on falls in production at peak incidence;

- if severe restrictions are imposed in response to the epidemic, without vaccination, accumulated falls in the value of production will reach over 3 years about $6.8-9.8 \%$ in a poorly developed economy or $6.7-9.6 \%$ in a strongly developed economy. If a liberal approach to the epidemic is adopted, the falls will reach $2.3-3.5 \%$ in a poor economy and $2.2-$ $3.3 \%$ in a wealthy economy;

- slow progress in immunization coverage of the population combined with severe restrictions imposed in response to the epidemic will lead to accumulated falls in production by $5.5-8.1 \%$ in a poorly developed economy or by $5.4-7.9 \%$ in a strongly developed economy. A liberal approach to the epidemic will lead to accumulated falls in production by $2.1-3.2 \%$ in a poor economy or $2.0-3.1 \%$ in a wealthy economy;

- a rapid pace of progress in immunization coverage of the population will reduce falls in production in a poor economy to $4.7-6.6 \%$ (severe

7 Those economies are also termed below "poor" and "wealthy". 
Modelling the impact of an epidemic 107

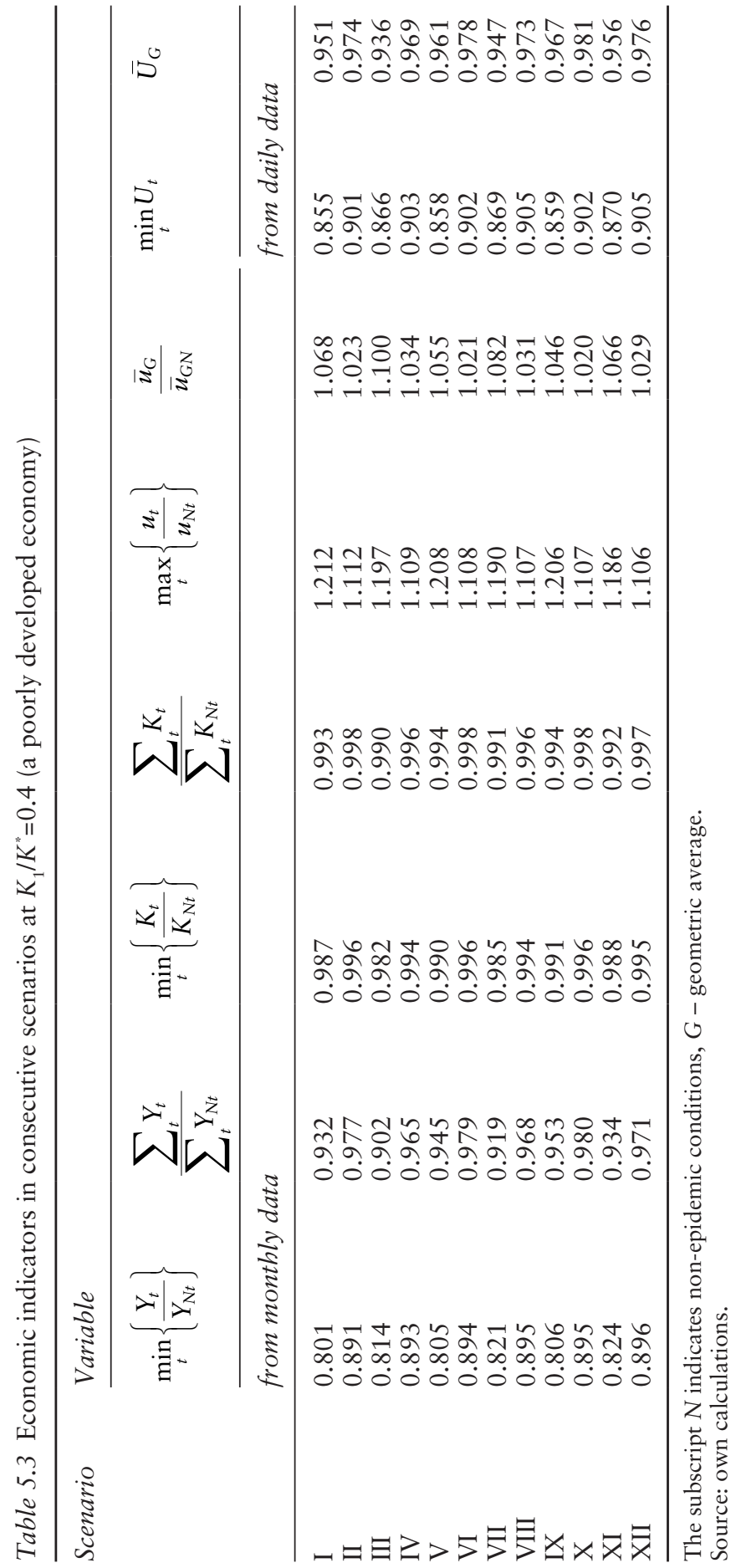




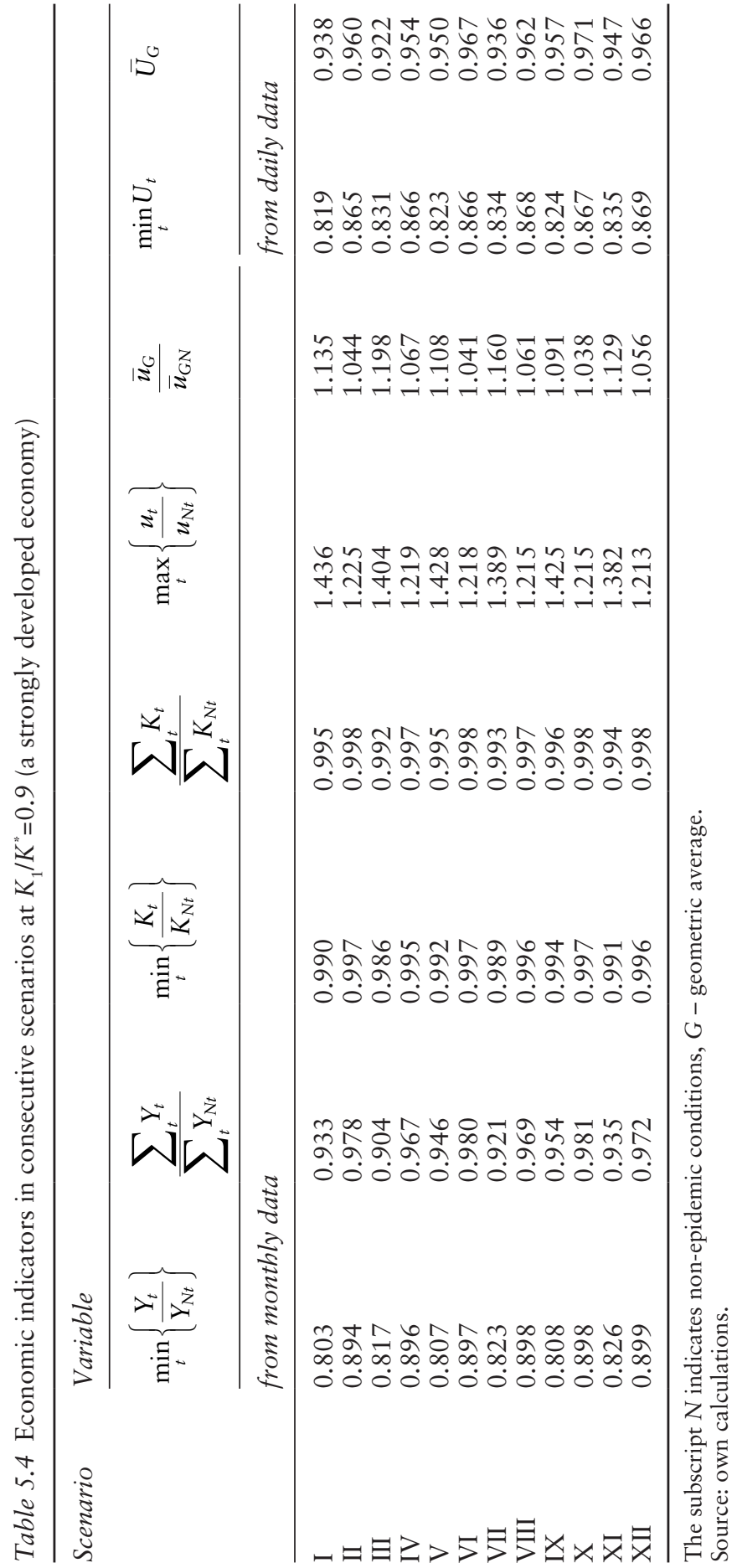




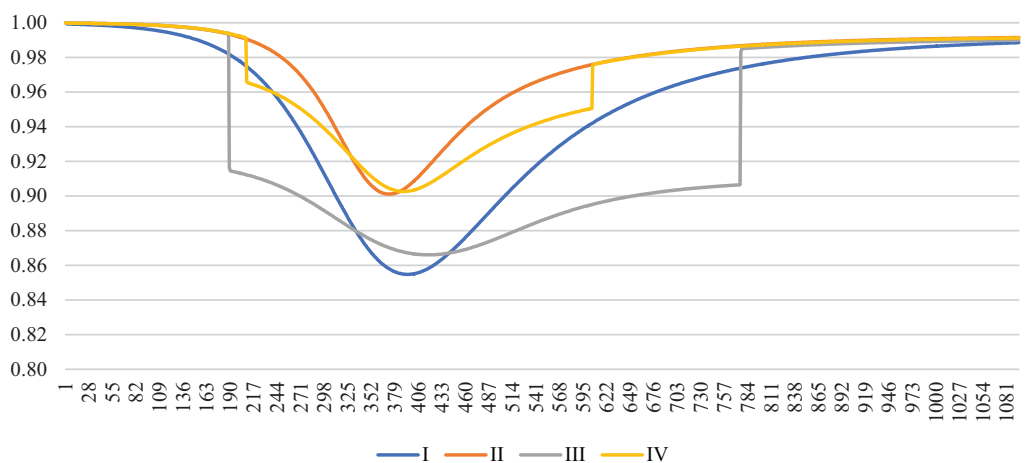

Figure 5.5a Curves representing social utility in scenarios I-IV. A poorly developed economy. Source: own calculations.

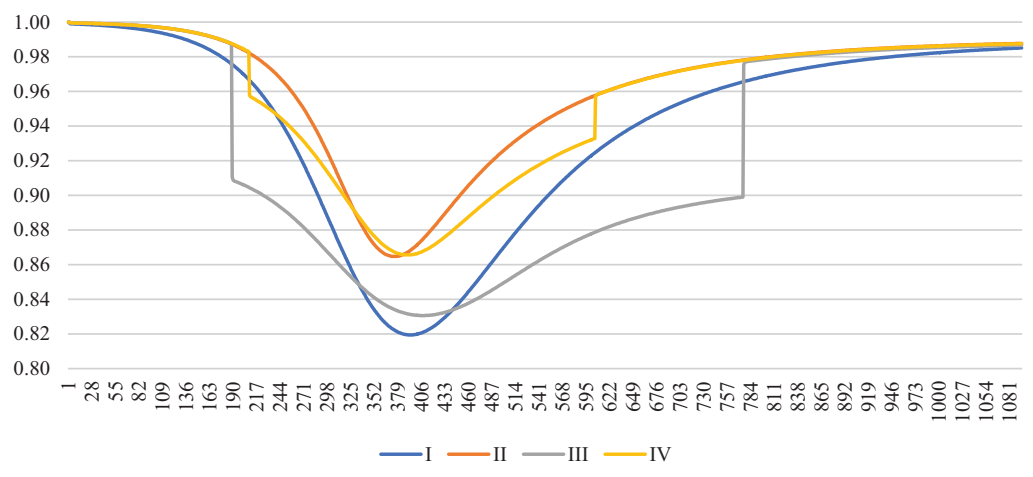

Figure 5.5b Curves representing social utility in scenarios I-IV. A strongly developed economy. Source: own calculations.

restrictions imposed) or 2.0-2.9\% (a liberal approach), and in a wealthy economy to $4.6-6.5 \%$ or $1.9-2.8 \%$;

- a more general conclusion can be reached: the introduction and rapid administration of a vaccine will have a stronger effect on accumulated falls in production than on the depth of the recession. In addition, both accumulated falls in production and the depth of recession will be slightly greater in a poorly developed economy than in a strongly developed economy;

- both one-off (at the epidemic peak) and accumulated falls in capital stock are significantly smaller than falls in production. Whether the government has access to a vaccine or not, whether severe restrictions are imposed or a liberal approach to the epidemic is adopted, accumulated falls in capital stock in both analyzed types of economy, that is $\frac{\sum_{t} K_{t}}{\sum_{t} K_{N t}}$, will not exceed $1 \%$; 


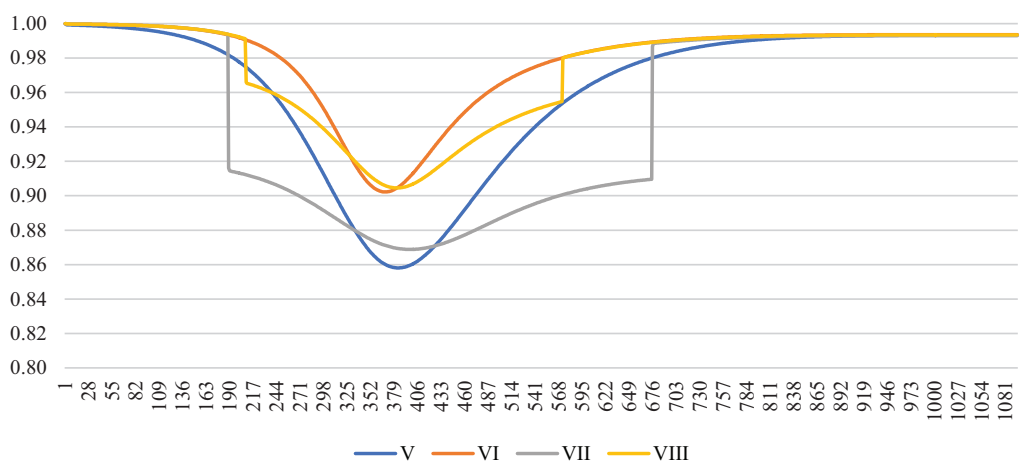

Figure 5.6a Curves representing social utility in scenarios V-VIII. A poorly developed economy. Source: own calculations.

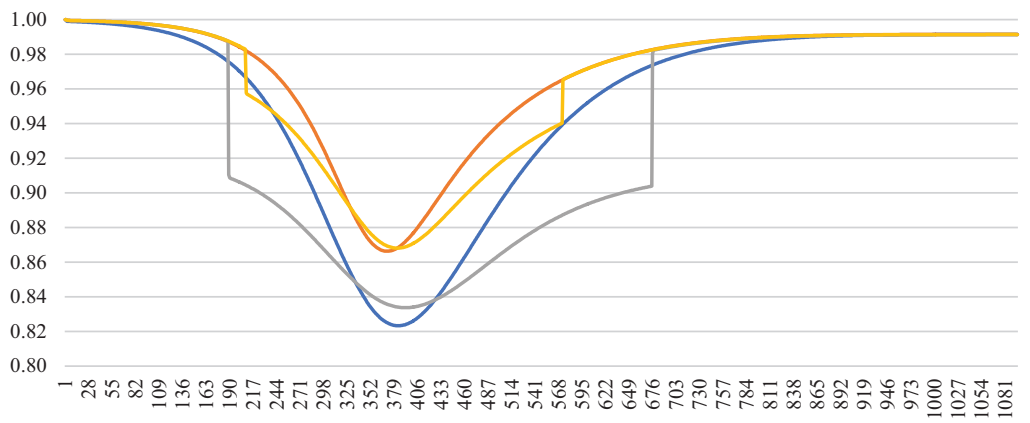

$$
-\mathrm{V}-\mathrm{VI}-\mathrm{VII}-\mathrm{VIII}
$$

Figure 5.6b Curves representing social utility in scenarios V-VIII. A strongly developed economy. Source: own calculations.

- relative increases in the unemployment rate (understood as $\max _{t}\left\{\frac{u_{t}}{u_{N t}}\right\}$ ) at peak incidence in a poor economy without vaccination will reach about $20 \%$ if severe restrictions are imposed in response to the epidemic, or about $10-11 \%$ if a liberal approach is adopted. In a wealthy economy the indicators will reach $40-44 \%$ or $22-23 \%{ }^{8}$

8 The parameters of the macroeconomic module of the proposed model are calibrated so that the initial unemployment rate in a poorly developed economy amounts to about $5 \%$, and in a strongly developed economy to about $10 \%$. Hence, the value of indicator $\max _{t}\left\{\frac{u_{t}}{u_{N t}}\right\}$ amounting e.g. to 1.1 means that the unemployment rate rises from $5 \%$ to $5.5 \%$ in a wealthy 


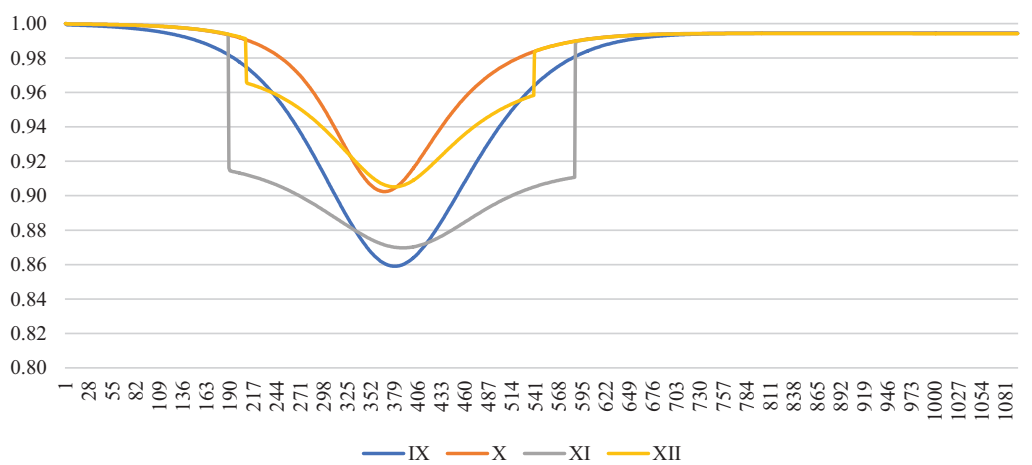

Figure $5.7 a$ Curves representing social utility in scenarios IX-XII. A poorly developed economy. Source: own calculations.

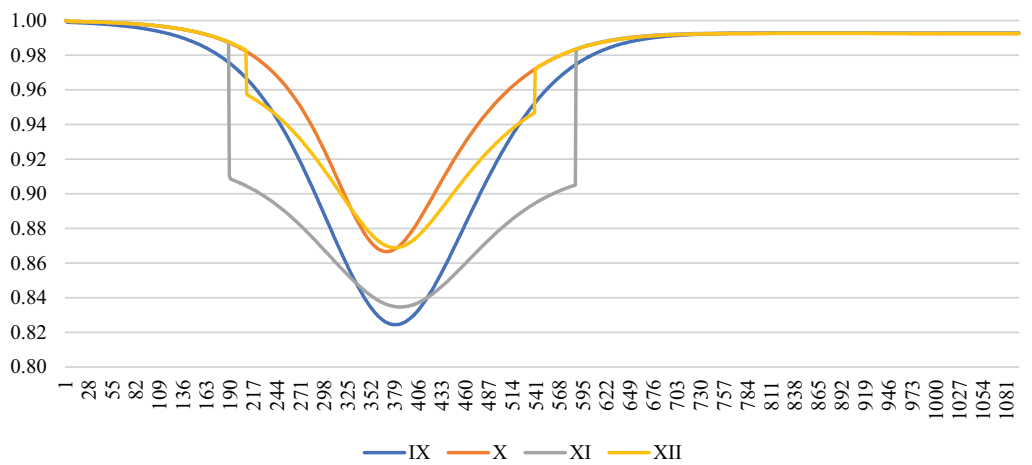

Figure 5.7b Curves representing social utility in scenarios IX-XII. A strongly developed economy. Source: own calculations.

- the indicators only slightly fall with slow or rapid progress in immunization coverage;

- the average unemployment rates over a three-year period (and more precisely the products $\frac{\bar{u}_{G}}{\bar{u}_{G N}}$ ) will be higher in the scenarios of severe restrictions imposed by the government in response to the epidemic and will decrease with an increase in the pace of immunization coverage of the population. Those products will also be higher in a wealthy economy. However, it must be emphasized that the geometric average of economy or from $10 \%$ to $11 \%$ in a poor economy. The indicator $\frac{\bar{u}_{G}}{\bar{u}_{G N}}$ is to be similarly
interpreted. 


\section{Monika Bolińska et al.}

the unemployment rate $\bar{u}_{G N}$ is significantly lower in a wealthy economy than in a poor economy due to the model design;

- Figures 5.5-5.7 (depicting curves of the social utility function in consecutive scenarios in a poor and in a wealthy economy) lead to the following conclusions. First, falls in social utility $U$ in both types of economy, in scenarios of severe restrictions imposed in response to the epidemic (the scenarios marked with odd Roman numerals) are significantly greater than in scenarios of a liberal approach (the scenarios marked with even numbers). Second, the sooner a vaccine is administered, the smaller are falls in social utility. Third, falls in social utility are slightly smaller in a poor economy, because expressions $\frac{u_{N t}}{u_{t}} \frac{Y_{t}}{Y_{N t}}$ are higher in
that type of economy.

\subsection{Conclusions}

Chapter 5 discusses the effect of an epidemic on economic growth. The analysis is conducted using a model of economic growth under epidemic conditions. The epidemiological module introduces an indicator that shows restrictions imposed on social and economic life during the epidemic. The indicator is defined in two versions; in the first version, it changes continually on consecutive days of the epidemic as a function of the percentage of infections, and in the second version, it changes discretely when the government abruptly imposes a lockdown. The epidemiological section also includes a scenario wherein a vaccine (against the spreading disease) is available to the government and a population vaccination programme is implemented. In the section of the model discussion that is dedicated to the economy, it is assumed that the production process is described by a neoclassical CobbDouglas production function; accumulation of fixed capital, like in the original Solow model of 1956, is defined as the difference between investment and the depreciated value of that capital. Also, a social utility function is introduced, defined as a geometrical average of the indicator of social and economic activity, the percentage of the uninfected, the ratio of unemployment rate under non-epidemic conditions to that rate under epidemic conditions and the ratio of production during the epidemic to production under non-epidemic conditions.

The chapter also discusses scenarios of epidemic development depending on the availability of a vaccine to the government. In the scenarios wherein the government has no access to a vaccine, it was assumed that the government imposes restrictions on social and economic activity following certain functional relations or abruptly. The scenarios with vaccination are divided into those with slow and those with rapid progress in immunization coverage of the population. Those scenarios also include a lockdown imposed by the government, like in the scenarios without vaccination.

Falls in production in an economy without access to a vaccine reach, at peak incidence, $18.3 \%$ to $19.9 \%$ if severe restrictions are imposed in response 
to the epidemic, or $10.4 \%$ to $10.9 \%$ if a liberal approach is adopted. Slow progress in immunization coverage of the population combined with severe restrictions imposed in response to the epidemic will reduce falls in production by $17.7 \%$ to $19.5 \%$. If a liberal approach to the epidemic is adopted, falls in production will reach (respectively) $10.2-10.6 \%$. Additionally, rapid progress in immunization coverage has no material effect on falls in production at peak incidence.

If the government imposes severe restrictions in response to the pandemic and has no access to a vaccine, accumulated falls in the value of production will reach, over three years, about $6.7-9.8 \%$, and if a liberal approach to the epidemic is adopted, the falls will reach $2.2-3.5 \%$. Slow progress in immunization coverage of the population combined with severe restrictions imposed in response to the pandemic will lead to accumulated falls in production of $5.4-8.1 \%$ while a liberal approach to the pandemic will lead to accumulated falls in production of $2.0-3.2 \%$. A rapid pace of progress in immunization coverage of the population reduces accumulated drops in production to about $4.6-6.6 \%$, if severe restrictions are imposed, or to $1.9-2.9 \%$. Consequently, the introduction of vaccination and rapid progress in immunization coverage will have a stronger effect on accumulated falls in production than on the depth of recession. Additionally, whether the government has access to a vaccine or not, falls in the capital stock will be significantly smaller than falls in production and will not exceed $1 \%$.

Relative increases in the unemployment rate at peak incidence in a poor economy without vaccination will reach about $20-44 \%$ if severe restrictions are imposed in response to the epidemic, or about $10-23 \%$ if a liberal approach is adopted. The introduction and acceleration of vaccination entails a minor reduction in relative rises in the unemployment rate at peak incidence. Additionally, average unemployment rates over a three-year period will be higher in the scenarios of severe restrictions imposed by the government in response to the epidemic and will decrease with an increase in the pace of immunization coverage of the population.

Falls in social utility will be significantly greater in scenarios of severe restrictions imposed in response to the epidemic than in scenarios of a liberal approach. Implementation of a vaccination programme will result in a reduced depth of fall in social utility, and the faster is progress in immunization coverage of the population, the relatively smaller are falls in social utility.

\section{References}

Alvarez, F., Argente, D., Lippi, F. (2020). A simple planning problem for COVID19 lockdown. NBER Working Paper, No. 26981. http:/www.nber.org/papers/ w26981 (accessed February 16, 2021).

Atkeson, A. (2020). What will be the economic impact of COVID-19 in the US? Rough estimates of disease scenarios. NBER Working Paper, No. 26867. http:// www.nber.org/papers/w26867 (accessed February 16, 2021). 


\section{Monika Bolińska et al.}

Aum, S., Lee, S., Shin, Y. (2020). Inequality of fear and self-quarantine: Is there a trade-off between GDP and public health? NBER Working Paper, No. 27100. http://www.nber.org/papers/w27100 (accessed February 16, 2021).

Bärwolff, G. (2020). Mathematical modeling and simulation of the COVID19 pandemic. Systems, 8(3), 1-12. https://doi.org/10.3390/systems8030024 (accessed February 16, 2021).

Bloom, D., Lyons, J. (Eds.) (1993). Economic implications of AIDS in Asia, United Nations Development Programme, New York.

Bloom, D., Mahal, A. (1995). Does the AIDS epidemic really threaten economic growth? National Bureau of Economic Research working paper No. 5148, NBER, Cambridge, MA. https://doi.org/10.1016/S0304-4076(96)01808-8 (accessed February 16, 2021).

Bloom, D., Mahal, A. (1997). Does the AIDS epidemic really threaten economic growth? Journal of Econometrics, 77(1), 105-124.

Brock, W., Xepapadeas, A. (2020). The economy, climate change and infectious diseases: Links and policy implications. Environmental and Resource Economics, 76, 811-824. https://doi.org/10.1007/s10640-020-00442-z (accessed February $16,2021)$.

Cuddington, J. (1993). Modeling the macroeconomic effects of AIDS, with an application to Tanzania. The World Bank Economic Review, 7, 173-789.

Cuddington, J., Hancock, J. (1994). Assessing the impact of aids on the growth path of the Malawian economy. Journal of Development Economics, 43(2), 363-368.

Cuesta, J. (2010). How much of a threat to economic growth is a mature AIDS epidemic? Applied Economics, 42(24), 3077-3089.

Delfino, D., Simmons, P. (2005). Dynamics of tuberculosis and economic growth. Environment and Development Economics, 10(6), 719-743. https://doi.org/10. 1017/S1355770X05002500 (accessed February 16, 2021).

Fei-Ying, Y., Wan-Tong, L., Zhi-Cheng, W. (2015). Traveling waves in a nonlocal dispersal SIR epidemic model. Nonlinear Analysis: Real World Applications, 23, 129-147.

Jardón-Kojakhmetov, H., Kuehn, Ch., Pugliese, A., Sensi, M. (2021). A geometric analysis of the SIR, SIRS and SIRWS epidemiological models. Nonlinear Analysis: Real World Applications, 58, 103220.

Kambou, G., Devarajan, S., Over, M. (1992). The economic impact of AIDS in an African country: Simulations with a computable general equilibrium model of Cameroon. Journal of African Economies, 1, 109-130.

Kermack, W. O., McKendrick, A. G. (1927). A contribution to the mathematical theory of epidemics. Royal Society, 115(772), 700-721.

Lik Ng, W. (2020). To lockdown? When to peak? Will there be an end? A macroeconomic analysis on COVID-19 epidemic in the United States. Journal of Macroeconomics, 65, 103230.

Lovasz, E., Schipp, B. (2009). The impact of HIV/AIDS on economic growth in SubSaharan Africa. South African Journal of Economics, 77(2), 245-256.

Mankiw, N. G., Romer, D., Weil, D. N. (1992). A contribution to the empirics of economic growth. Quarterly Journal of Economics, 107(2), 407-437.

Murray, J. D. (2003). Mathematical biology, II, spatial models and biomedical applications, in Interdisciplinary applied mathematics, 3rd ed., vol. 18, SpringerVerlag, New York. 
Ruan, S. (2007). Spatial-temporal dynamics in nonlocal epidemiological models, in Y. Takeuchi, K. Sato, Y. Iwasa (Eds.), Mathematics for life science and medicine, Springer-Verlag, Berlin, pp. 99-122.

Solow, R. M. (1956). A contribution to the theory of economic growth, Quarterly Journal of Economics, 70(1), 65-94.

Xiao, D., Ruan, S. (2007). Global analysis of an epidemic model with nonmonotone incidence rate, Mathematical Biosciences, 208, 419-429. 